\title{
Finsler Streamline Tracking with Single Tensor Orientation Distribution Function for High Angular Resolution Diffusion Imaging
}

\author{
Laura Astola $\cdot$ Andrei Jalba $\cdot$ Evgeniya Balmashnova • \\ Luc Florack
}

Published online: 11 February 2011

(C) The Author(s) 2011. This article is published with open access at Springerlink.com

\begin{abstract}
We introduce a new framework based on Riemann-Finsler geometry for the analysis of 3D images with spherical codomain, more precisely, for which each voxel contains a set of directional measurements represented as samples on the unit sphere (antipodal points identified). The application we consider here is in medical imaging, notably in High Angular Resolution Diffusion Imaging (HARDI), but the methods are general and can be applied also in other contexts, such as material science, et cetera, whenever direction dependent quantities are relevant. Finding neural axons in human brain white matter is of significant importance in understanding human neurophysiology, and the possibility to extract them from a HARDI image has a potentially major impact on clinical practice, such as in neuronavigation, deep brain stimulation, et cetera. In this paper we introduce a novel fiber tracking method which is a generalization of the streamline tracking used extensively in Diffusion Tensor Imaging (DTI). This method is capable of finding intersecting fibers in voxels with complex diffusion
\end{abstract}

L. Astola $(\bowtie)$

Biometris, Plant Science Group, Wageningen University \& Research Centre, Droevendaalsesteeg 1 Building 107, 6708 PB Wageningen, The Netherlands

e-mail: laura.astola@wur.nl

A. Jalba $\cdot$ E. Balmashnova $\cdot$ L. Florack

Department of Mathematics and Computer Science, Eindhoven University of Technology, Den Dolech 2, 5612AZ Eindhoven, The Netherlands
A. Jalba
e-mail: a.c.jalba@tue.nl
E. Balmashnova
e-mail: e.balmachnova@tue.nl
L. Florack
e-mail:1.m.j.florack@tue.nl

profiles, and does not involve solving extrema of these profiles. We also introduce a single tensor representation for the orientation distribution function (ODF) to model the probability that a vector corresponds to a tangent of a fiber. The single tensor representation is chosen because it allows a natural choice of Finsler norm as well as regularization via the Laplace-Beltrami operator. In addition we define a new connectivity measure for HARDI-curves to filter the most prominent fiber candidates. We show some very promising results on both synthetic and real data.

Keywords ODF · DTI · HARDI · Riemann geometry · Finsler geometry $\cdot$ Fiber tracking

\section{Introduction}

Diffusion Tensor Imaging (DTI) is a non-invasive magnetic resonance imaging modality to measure molecular motion of water in biological tissue. It is primarily used to image muscular tissue and tissue consisting of densely packed neural axons such as brain white matter. A typical voxel size is $1 \mathrm{~mm}^{3}$, while a typical diameter of a single neural axon is in the order of micrometers. An important challenge is to extract the axonal architecture and to find the fiber bundles (tractography) connecting different regions of the brain (connectivity). It is assumed that the local diffusion profile is indicative of the gross fiber orientation distribution in a voxel, since there is more diffusion along the direction of elongation of axons than across these structures.

The DTI model yields an ellipsoidal profile as the level set of the water diffusivity profile after finite-time diffusion. Such a profile predicts the fiber directions well only in case the voxel contains fibers with a single preferred direction. As a symmetric and positive definite (SPD) tensor, the diffusion 
tensor (or its inverse) can be interpreted as a Riemann metric tensor. For this reason several authors have applied tools from Riemannian geometry to the analysis of DTI data [4, 6, 17, 21, 30].

High Angular Resolution Diffusion Imaging (HARDI)is a collective name for techniques that capture more directional measurements, improving the angular resolution. HARDI requires a model of local diffusion that is more complex than the rank-two DTI tensor model [24, 35, 39]. Finsler geometry can incorporate more general local norm functions than one induced by a Riemannian inner product and is indeed applicable to the analysis of HARDI data [5, 27].

From the various HARDI models we adopt here the socalled Q-ball imaging technique $[3,15,36]$ and model HARDI measurements using spherical tensors, i.e. homogeneous polynomials restricted to the sphere. However, our method is by no means restricted to Q-ball imaging. Recently more advanced methods to reconstruct the ODF or the so-called orientation probability density function have emerged $[2,34]$ and our method is applicable to these as well.

Using a tensor representation, one can construct a Finsler norm suitable for the analysis of diffusion profiles that can have a more general shape than that of an ellipsoid. On the other hand, to regularize noisy HARDI data based on heat diffusion on the sphere, we need a decomposition of the tensor into homogeneous and harmonic components that are the eigenfunctions of the Laplace-Beltrami operator [18, 29]. We show how to do this in one step without iteratively fitting different order harmonics. We also introduce a novel method for fiber tracking in HARDI data based on Finsler geometry. The idea of Finsler geometry in a HARDI setting has been proposed by Melonakos et al. [27], who adopted a dynamic programming approach to compute optimal curves with respect to a Finsler norm, without computing Finsler metric tensors and associated eigensystems as we do below. In contrast to their work, we use a tensor model for which the metric can be computed a priori, ODE-based tracking, different homogenization technique, and explicit Tikhonov regularization based on Laplace-Beltrami diffusion. Regularization is critical, since HARDI data tend to have low SNR.

This paper is organized as follows: In Sect. 2 we show how to compute the regularized orientation distribution function (ODF) in a tensor framework. In Sect. 3 we compute Finslerian diffusion tensors from this ODF. In Sect. 4 we extend DTI-streamline tracking to Finslerian streamline tracking for HARDI data, to allow crossings of fibers. Finally in Sect. 5 we show some promising experimental results on both simulated and real HARDI data.

\section{Orientation Distribution Function on a Spherical Tensor Field}

In HARDI literature spherical harmonics are a natural and popular choice to represent functions on the sphere [15, 23]. Nevertheless, in this paper we prefer an equivalent tensorisomorphic to homogeneous polynomial-representation $[18,38]$, since such a description is more natural in a differential geometric approach.

A (covariant) tensor $D$ of degree $n$ at a point $p$ on a differentiable manifold $M$ can be seen as a multilinear mapping

$D(p): \underbrace{T_{p} M \times \cdots \times T_{p} M}_{n \text { times }} \rightarrow \mathbb{R}$

in which $T_{p} M$ denotes the tangent space at $p \in M$ [26]. The point $p$ has coordinates $x=\left(x^{1}, x^{2}, x^{3}\right) \in \mathbb{R}^{3}$ relative to a local coordinate chart, but explicit reference to this point will often be suppressed for ease of notation. All directionally dependent functions used below depend on this position $x$, and on a local direction vector $y \in \mathbb{R}^{3}$. The vector $y$ may or may not be confined to the unit sphere (it should be clear from the context if a constraint is applicable). In the latter case it may be expressed in spherical coordinates $\theta$ and $\varphi$, but it is tacitly understood that in this case antipodal points are to be identified (implying that the functions under consideration are symmetrical).

For example, a second order symmetric spherical tensor in $\mathbb{R}^{3}$ can be written as

$$
\begin{aligned}
D_{i j} y^{i} y^{j}= & D_{11} y^{1} y^{1}+D_{12} y^{1} y^{2}+D_{13} y^{1} y^{3} \\
& +D_{21} y^{2} y^{1}+D_{22} y^{2} y^{2}+D_{23} y^{2} y^{3} \\
& +D_{31} y^{3} y^{1}+D_{32} y^{3} y^{2}+D_{33} y^{3} y^{3} \\
= & D_{11} y^{1} y^{1}+2 D_{12} y^{1} y^{2}+2 D_{13} y^{1} y^{3} \\
& +D_{22} y^{2} y^{2}+2 D_{23} y^{2} y^{3}+D_{33} y^{3} y^{3}
\end{aligned}
$$

suppressing the fiducial point $p$ in the notation and defining

$$
\begin{aligned}
y & =\left(y^{1}, y^{2}, y^{3}\right) \\
& =(\sin \theta \cos \varphi, \sin \theta \sin \varphi, \cos \theta),
\end{aligned}
$$

with the following summation convention

$a_{i} b^{i}:=\sum_{i} a_{i} b^{i}$.

To justify the choice we recall that spherical harmonics are in fact homogeneous harmonic polynomials restricted to unit sphere [29]. On the other hand, the choice of using tensors instead of equivalent homogeneous polynomials makes some operations elementary and intuitive. This is especially 
true for our case where only even order symmetric tensors are involved. A symmetric tensor here means that

$D_{i_{1} \cdots i_{n}}=D_{\sigma\left(i_{1} \cdots i_{n}\right)}$,

for any index permutation $\sigma$.

In Q-ball imaging the diffusion profile $\Psi$ is obtained as

$\Psi(y)=\int_{0}^{\infty} P(r y) d r$,

where $P(r y)$ is the ensemble-average probability that a particle is displaced from a fiducial initial point $x_{0}$ (implicit in the notation) to $x_{0}+r y$. In [11] it is shown that assuming short diffusion gradient pulses in the scanning protocol, the relation between signal $S(q)$ and $P(r)$ is

$P(r)=\int_{\mathbb{R}^{3}} S(q) e^{i 2 \pi r \cdot q} d q$,

where $q$ is the wave vector i.e. a unit vector encoding the direction and pulse duration. Using this relation, in [35] it is further shown that $\Psi$ in (6) can be estimated by the FunkRadon transform of the Fourier transform of $P(r)$. In [15] this result is applied to the case that the signal is modeled with spherical harmonics and it is shown that the ODF on a single shell can be approximated as

$\left.\Psi(y)\right|_{r=1} \approx 2 \pi \sum_{\ell=0}^{n} \sum_{m=-\ell}^{\ell} c_{\ell m} Y_{m}^{\ell}(y) P_{\ell}(0)$,

where $P_{\ell}$ denotes the Legendre polynomial of degree $\ell$. In particular we have

$P_{\ell}(0)= \begin{cases}0 & \ell \text { odd } \\ (-1)^{\ell / 2 \frac{1 \cdot 3 \cdot 5 \cdots(\ell-1)}{2 \cdot 4 \cdot 6 \cdots \ell}} & \ell \text { even. }\end{cases}$

The main benefit of this approach is that one can compute the ODF in a fast and robust way using spherical harmonics $(\mathrm{SH})$, and also apply Laplace-Beltrami regularization (to any order), which is a generalization of Gaussian smoothing for $\mathbb{L}_{2}$ functions on $\mathbb{R}^{2}$ to $\mathbb{L}_{2}$ functions on the sphere $\mathbb{S}^{2}$ [10].

We use this result, but replace the spherical harmonics by a tensor, cf. [38], which is more practical for applications using Finsler geometry.

We remark that recently a more accurate approach for ODF reconstruction has been given by Aganj et al. in [2]. However the tensor approach in this paper does not depend on the method with which the ODF is built. As long as there is an analytical expression for the ODF, we can construct regularized Finsler norms and metrics essential for the Finslerian fiber tracking.

For comparison we recall the procedure of fitting spherical harmonics to HARDI data according to [15] and applying Laplace-Beltrami diffusion on the harmonic components [16].
This is done as follows.

1. A set of real bases $\left\{Y_{i}\right\}_{i=1}^{N}$ of spherical harmonics up to the desired order is fitted to data sampled on the sphere $\left\{S\left(\theta_{k}, \varphi_{k}\right) \mid k=1, \ldots, m\right\}$ using least squares:

$S(\theta, \varphi)=\sum_{i=1}^{N} c_{i} Y_{i}(\theta, \varphi)$

Here the index $i$ collectively denotes the indexed pair $(\ell, m)$ as used in (8), partially ordered along incremental $\ell$-values. The order $\ell$ for a given index $i$ will henceforth be denoted by $\ell_{i}$.

2. The signal is regularized using Laplace-Beltrami diffusion $[16,18,19]$ :

$$
S(\theta, \varphi, \tau)=\sum_{i=1}^{N} e^{-\tau \ell_{i}\left(\ell_{i}+1\right)} c_{i} Y_{i}(\theta, \varphi) .
$$

3. Each spherical harmonic $Y_{i}$ of order $\ell_{i}$ is multiplied by the factor $2 \pi P_{\ell_{i}}(0)$.

4. Then

$$
\operatorname{ODF}(\theta, \varphi, \tau):=\sum_{i=1}^{N} 2 \pi P_{\ell_{i}}(0) e^{-\tau \ell_{i}\left(\ell_{i}+1\right)} c_{i} Y_{i}(\theta, \varphi) .
$$

Returning to our tensor approach, the principle is the same as in the recipe above, but in addition this approach allows us to construct Finsler norms related to the local diffusivity. Moreover, it does not require reference to or storage of spherical harmonics. In this approach the previous steps are replaced by the following:

1. We fit a $n$th order tensor $D$ (recall that $n$ is always even) to the sampled data on the sphere:

$S(y)=D(y)=D_{i_{1} \cdots i_{n}} y^{i_{1}} \cdots y^{i_{n}}$,

using the linear least squares. For details of the fitting procedure one may consult [14]. This is equivalent to fitting a polynomial consisting of $n$th order monomials to the data in such a way that the error at measured points is minimal in $\mathbb{L}_{2}\left(\mathbb{S}^{2}\right)$ norm. To ensure that fourth-order tensor is always positive definite we can make use of a method suggested by Barmpoutis et al. in [9] and improved in [20]. For higher order tensors we may apply the PSDT (positive semidefinite diffusion tensor) model of Qi et al. in [32].

2. From the tensor $D$ we compute the harmonic components $H_{n}, H_{n-2}, \ldots, H_{0}$, for which

$$
\triangle^{k / 2} H_{k}(y)=0, \quad k=n, n-2, \ldots, 0,
$$


where $\triangle$ is the standard Laplace operator, and decompose $D$ as follows:

$$
D(y)=\sum_{k=0}^{n} H_{k}(y)\left(\left(y^{1}\right)^{2}+\left(y^{2}\right)^{2}+\left(y^{3}\right)^{2}\right)^{(n-k)}
$$

where when evaluating this function, we can simplify the last element in each summand since

$$
\left(\left(y^{1}\right)^{2}+\left(y^{2}\right)^{2}+\left(y^{3}\right)^{2}\right)^{(n-k)}=1,
$$

for $y$ is a unit vector. The harmonic components $H_{k}(y)$ are in fact eigenfunctions of the Laplace-Beltrami operator on the sphere, with eigenvalues $k(k+1)$ [29]. This means that a harmonic component $H_{k}$ is actually a linear combination of spherical harmonics $Y_{m}^{k}$ and can be regularized in the same manner.

This allows us to use the results for Q-ball imaging $[15,36]$. The harmonic components can be easily computed using the so-called Clebsch-projection [29]. We have included a simple example on how to compute harmonic components of a second order polynomial in the Appendix. The extension to higher even order polynomials is straightforward.

3. Laplace-Beltrami regularization can be applied to each $H_{k}(y)$ :

$$
D(y, \tau)=\sum_{k=0}^{n} e^{-k(k+1) \tau} H_{k}(y) .
$$

4. Similar to (12) we now have

$$
\operatorname{ODF}(y, \tau):=\sum_{k=0}^{n} 2 \pi P_{\ell_{k}}(0) e^{-k(k+1) \tau} H_{k}(y) .
$$

Thus even the knowledge of a basis of real spherical harmonics is not needed. We remark that an $n$th order symmetric tensor in dimension three has

$N_{o}=\left(\begin{array}{c}n+2 \\ n\end{array}\right)=\frac{(n+2)(n+1)}{2}$

independent terms. Thus an $n$th order tensor is determined by $N_{o}$ numbers. The Clebsch decomposition can be computed very fast, since all the coefficients in harmonic components are linear combinations of the $N_{o}$ terms in the original tensor. This means that we can compute the components of the ODF-tensor with a regularization parameter $\tau$, by simply multiplying the components of the signal-tensor by a matrix. We give an explicit example of this in the Appendix.

Now that we have a tensor representation of the local diffusion probability, we use it to construct generalized, Finsler diffusion tensors. From these one can compute principal eigenvectors, since at each point and to every tangent vector there is a corresponding second order tensor approximating the local diffusivity, as we shall see in the following section.
Fig. 1 (Color online) Top: A fourth order tensor fitted to a sample of measurements (red dots). Bottom: For a given direction vector (here green and blue) we have an ellipsoid with corresponding color that approximates the diffusion profile
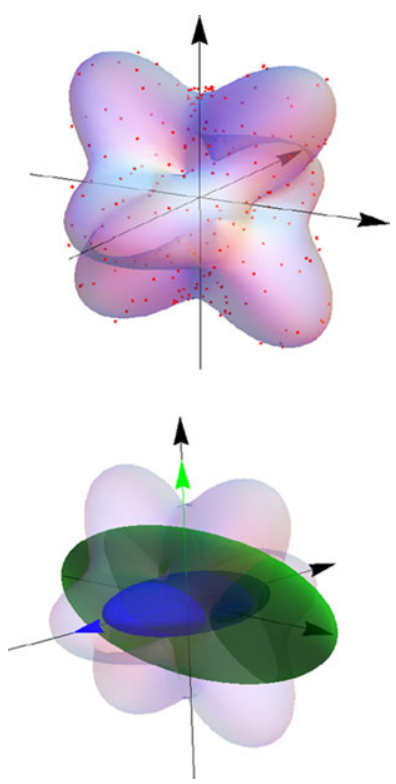

\section{From Orientation Distribution Function to Metric}

Riemannian geometry extends analytical geometry to curved spaces and equips these with a local inner product, which in turn allows computations of lengths and angles. Riemannian distances thus generalize the theorem of Pythagoras into skew coordinates. A norm function induced by a Riemannian inner product is a special case of a Finsler norm function [8, 12, 33]. A Finsler norm depends on both position and direction. A general convex norm can capture more complex angular dependence than the Riemann metric tensor. For an illustration, see Fig. 1.

In [5] it is shown that a Finsler structure can be defined on a HARDI image and the details of computing the metric/diffusion tensor from HARDI-ODF function are described. To make this paper self-contained, we repeat here the algorithm to compute generalized diffusion tensors $g(x, y)$ from the raw HARDI signal $S(x, y)$ :

1. The starting point is a single $n$th order tensor $\tilde{F}(x, y)$ representing the ODF. We obtain a homogeneous function $F(x, y)$ as follows ${ }^{1}$

$$
F(x, y)=\tilde{F}(x, y)^{1 / n}=\left(\tilde{F}_{i_{1} \cdots i_{n}}(x) y^{i_{1}} \cdots y^{i_{n}}\right)^{1 / n}
$$

2. When $F(x, \cdot)$ is strongly convex (true with typical HARDI data), we obtain a symmetric positive definite tensor:

$$
g_{i j}(x, y)=\frac{1}{2} \frac{\partial F^{2}(x, y)}{\partial y^{i} \partial y^{j}} .
$$

\footnotetext{
${ }^{1}$ Here homogeneity means $F(x, \lambda y)=\lambda F(x, y)$. Recall also that $\tilde{F}(x, y)$ is non-negative for all $x, y$.
} 
In case the norm $F$ is a square root of an inner product (Riemann metric), then $g=F$.

We have here two distinct heuristic interpretations for our applications. When applying Finsler geometry, we deal with norms and inner products that define distances. Then it is intuitive to replace ODF with the multiplicative inverse of ODF in all computations, for larger diffusivity implies shorter travel time from the diffusing particle point of view. Since water molecules in human tissue have constant average velocity $v$, the direct proportionality of (tissue structure induced) distance and diffusion time can be deduced trivially from $\Delta x=v \Delta t$.

On the other hand, when we want to work directly with the probabilistic diffusion profile and generalize the streamline tracking to HARDI case, we adopt directly the convention $\tilde{F}=\mathrm{ODF}$, and compute the direction dependent diffusion tensor as in (21). This diffusion tensor describes a quadratic form corresponding to small perturbations of $y$, which can be seen also from the following second order Taylor expansion of $F^{2}$ with fixed $x$ (suppressed in the notation henceforth). Consider the one-dimensional case for simplicity, and denote $F^{\prime}=\frac{d}{d y} F$, then

$$
\begin{aligned}
& F^{2}(y+\delta) \\
& \quad=F^{2}(y)+\left(F^{2}(y)\right)^{\prime} \delta+\frac{1}{2}\left(F^{2}(y)\right)^{\prime \prime} \delta^{2}+\mathcal{O}\left(\delta^{3}\right) \\
& \approx F^{2}(y)+2 F(y) F^{\prime}(y) \delta+\frac{1}{2}\left(F^{2}(y)\right)^{\prime \prime} \delta^{2} .
\end{aligned}
$$

Using also the fact that

$$
F(y+\delta)=F(y)+F^{\prime}(y) \delta+\mathcal{O}\left(\delta^{2}\right),
$$

we get

$$
\frac{1}{2}\left(F^{2}(y)\right)^{\prime \prime} \approx \frac{(F(y+\delta)-F(\delta))^{2}}{\delta^{2}} .
$$

\section{Finslerian Streamlines}

\subsection{Background}

The most popular way to track axon(bundle)-like curves in DTI data is to follow the principal eigenvectors of diffusion tensors $D^{i j}(x)$. This approach requires that the curve $c:[0,1] \rightarrow \mathbb{R}^{3}$ satisfies the following equations

$$
\left\{\begin{array}{l}
\dot{c}(t)=\arg \max _{|h|=1}\left\{D^{i j}(c(t)) h_{i} h_{j}\right\} \\
c(0)=p \\
\dot{c}(0)=V .
\end{array}\right.
$$

The solution to this equation is a geodesic from a point $p$ to a sphere $\mathbb{S}^{2}(p, \varepsilon)$ for some small $\varepsilon$, but not necessarily a
Fig. 2 Left: A second order tessellation gives the equiangular unit vectors used here as initial directions for fiber tracking at the initial point/voxel

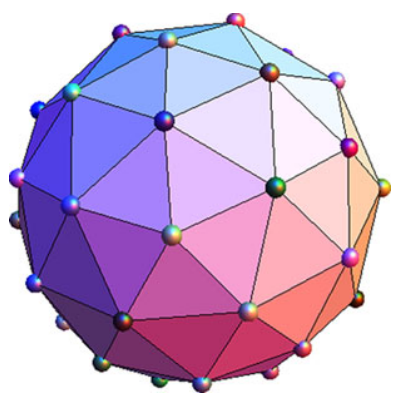

geodesic between $c(0)$ and $c(1)$. This tracking scheme can be extended to HARDI framework using Finsler geometry by simply replacing $D^{i j}(c(t))$ with $D^{i j}(c(t), \dot{c}(t))$, where

$\dot{c}(t)=\lim _{\delta \rightarrow 0} \frac{c(t)-c(t-\delta)}{\delta}$.

\subsection{Algorithm}

Our tracking algorithm belongs to the class of the so-called fiber assignment by continuous tracking (FACT) algorithms [28]. The practical implementation is similar to that of the streamline tracking in DTI setting. One difference is that since the diffusion profile is not ellipsoidal, but (potentially much) more complex, so that there is no unique initial principal eigenvector at the initial point of tracking. Instead we start tracking in all gradient directions. The user can define the gradients to e.g. correspond to the points of a tessellation of any order of choice. Here we have used the second order tessellation (zeroth order being the icosahedron) with 54 gradients (see Fig. 2).

Another difference is that there is no unique second order local diffusion tensor per point. Instead there is a unique second order local diffusion tensor corresponding given the direction of arrival (26) at a given point. This local diffusion tensor is computed as in (21) in Sect. 3. The formula for the appropriate local diffusion tensor is easily computed beforehand. For example, if we have a fourth order tensor,

$D_{4}(x, y):=D_{i j k l}(x) y^{i} y^{j} y^{k} y^{l}$

describing the diffusion profile, the Finsler bi-linear form (21) corresponding to the homogenized form

$F(x, y):=\left(D_{4}(x, y)\right)^{1 / 4}$

is

$$
\begin{aligned}
& g_{i j}(x, y) \\
& =\frac{1}{2} \frac{\partial^{2} F^{2}(x, y)}{\partial y^{i} \partial y^{j}} \\
& =\frac{1}{2} \frac{\partial^{2} D_{4}^{1 / 2}}{\partial y^{i} \partial y^{j}}
\end{aligned}
$$


Table 1 Comparison of the algorithmic structure between the proposed Finsler streamline tracking, DTI-streamline tracking and a method that follows the maxima of the ODF

\begin{tabular}{lll}
\hline DTI/Riemann & HARDI/maxima & HARDI/Finsler \\
\hline & find maxima of & compute Hessian \\
& ODF & $\begin{array}{l}\text { of ODF } \\
\text { find eigensystem generalized }\end{array}$ \\
find eigensystem & & diffusion tensor \\
diffusion tensor & & follow principal \\
follow principal & follow maxima \\
eigenvector & & eigenvector \\
\hline
\end{tabular}

$$
\begin{aligned}
= & 3 D_{4}^{-1 / 2} D_{i j q p}(x) y^{q} y^{p} \\
& -2 D_{4}^{-3 / 2} D_{i p k l}(x) y^{p} y^{k} y^{l} D_{q j m n}(x) y^{q} y^{m} y^{n},
\end{aligned}
$$

where we have put $D_{4}:=D_{4}(x, y)$ for brevity.

Now we have a bi-linear form at every position so that if we supply the vector $y$ of the direction of arrival, it returns a local diffusion tensor.

If the direction of arrival is close to the principal eigenvector of this local diffusion tensor, we take a step forward, if not we stop. The familiar anisotropy criterion for DTI, which stops tracking if the diffusion tensor is isotropic, can be applied to this local diffusion tensor as well.

Thus in a nutshell the algorithm is as follows: If the tangent of the streamline is in reasonable alignment with the principal eigenvector defined by the local diffusion tensor (corresponding to the tangent) and the fractional anisotropy of the local diffusion tensor is high enough, proceed, otherwise stop. A comparison between the essential steps in Finsler streamline tracking, DTI-streamline tracking and a maxima extraction method is summarized in Table 1 . The computation of a diffusion tensor or ODF (in terms of spherical harmonics or equivalently in terms of a monomial tensor) is considered as a preprocessing step and is not included in the table.

The only difference between our method and the standard DTI streamline tracking is the extra step in which the Hessian of the ODF is computed. However, in Sect. 5.3 we show that this additional step is relatively inexpensive and can be performed with less overhead. On the other hand, standard ODF-based tracking methods [13] typically require finding multiple, local maxima of the ODF. This in turn can be expensive in terms of computations [1]. For example, one can attempt to find all the local maxima of the ODF by numerical, iterative approaches, e.g. Newton method. However, such an approach would also require an additional exhaustive, two-dimensional search, to guarantee that all maxima are found, with accuracy within the discretization resolution. Thus, the complexity of such an attempt would be quadratic in the desired accuracy. Although, it is possible to reduce the search space to one dimension [1], the computation of all local maxima of the ODF remains an expensive operation. Moreover, if the streamline tracking algorithm is required to follow all local maxima, branching (or splitting) of the streamlines has to be allowed [13], which further increases the computational requirements. Finally, the simple stopping criterion based on the computation of the fractional anisotropy (FA), now relies on the more computationally involved, generalized anisotropy measure [35, 40].

In Sect. 5.3 we show that even if only one ODF (global) maximum is used for advancing the streamline, the resulting method is still more expensive than ours. Note that, to find the global maximum we simply evaluate the ODF in a number of search directions (i.e. scanning directions) and deem the largest value as the sought maximum. Clearly such a naive approach can potentially be very inaccurate, yet it is certainly faster than both the iterative approach and the method in [1].

\subsection{Connectivity measure}

The connectivity measure for an arbitrary curve defined here indicates the relative amount of diffusivity along this curve. Higher value implies greater diffusivity. In isotropic regions its value is one. In DTI setting, to compute the so-called connectivity [7,31] of a curve (fiber-candidate), we compare the Euclidean length of the curve to its Riemannian length (determined by the diffusion tensors along the curve) and define a connectivity measure $m(\gamma)$ of a curve $\gamma(t)$ as follows:

$m(\gamma)=\frac{\int \sqrt{\eta_{i j}(\gamma(t)) \dot{\gamma}(t)^{i} \dot{\gamma}(t)^{j}} d t}{\int \sqrt{g_{k l}(\gamma(t)) \dot{\gamma}(t)^{k} \dot{\gamma}(t)^{l}} d t}$,

where the $\eta_{i j}(\gamma)$ represents the covariant Euclidean metric tensor, which in Cartesian coordinates reduces to the constant identity matrix, $\dot{\gamma}$ the tangent to the curve $\gamma$ and $g_{k l}(\gamma)$ the Riemann-metric tensor.

We can generalize this connectivity measure directly to HARDI setting.

$m(\gamma)=\frac{\int \sqrt{\eta_{i j}(\gamma(t)) \dot{\gamma}(t)^{i} \dot{\gamma}(t)^{j}} d t}{\int \sqrt{g_{k l}(\gamma(t), \dot{\gamma}(t)) \dot{\gamma}(t)^{k} \dot{\gamma}(t)^{l}} d t}$,

where $g_{k l}(\gamma, \dot{\gamma})$ is the Finsler-metric tensor (which depends not only on the position but also on the direction of the curve). Since $g_{i j}(x, y) y^{i} y^{j}=F^{2}(x, y)$, this can be simplified to

$m(\gamma)=\frac{\int \sqrt{\eta_{i j}(\gamma(t)) \dot{\gamma}(t)^{i} \dot{\gamma}(t)^{j}} d t}{\int F(\gamma(t), \dot{\gamma}(t)) d t}$.

This measure can be computed along any curve. In Sect. 5 we show results of fiber tracking and connectivity measures. 
Fig. 3 (Color online) Left: Streamlines in a noise free tensor field simulating a $65^{\circ}$ crossing of fiber bundles. Streamlines are colored according to their connectivity. High (low) connectivity is color coded as red (blue). Right: Same field with Rician noise added

Fig. 4 Left: Streamlines in a noise free tensor field simulating a $30^{\circ}$ crossing of fiber bundles. Note that smaller angle results in kissing streamlines. Right: Same field with Rician noise added
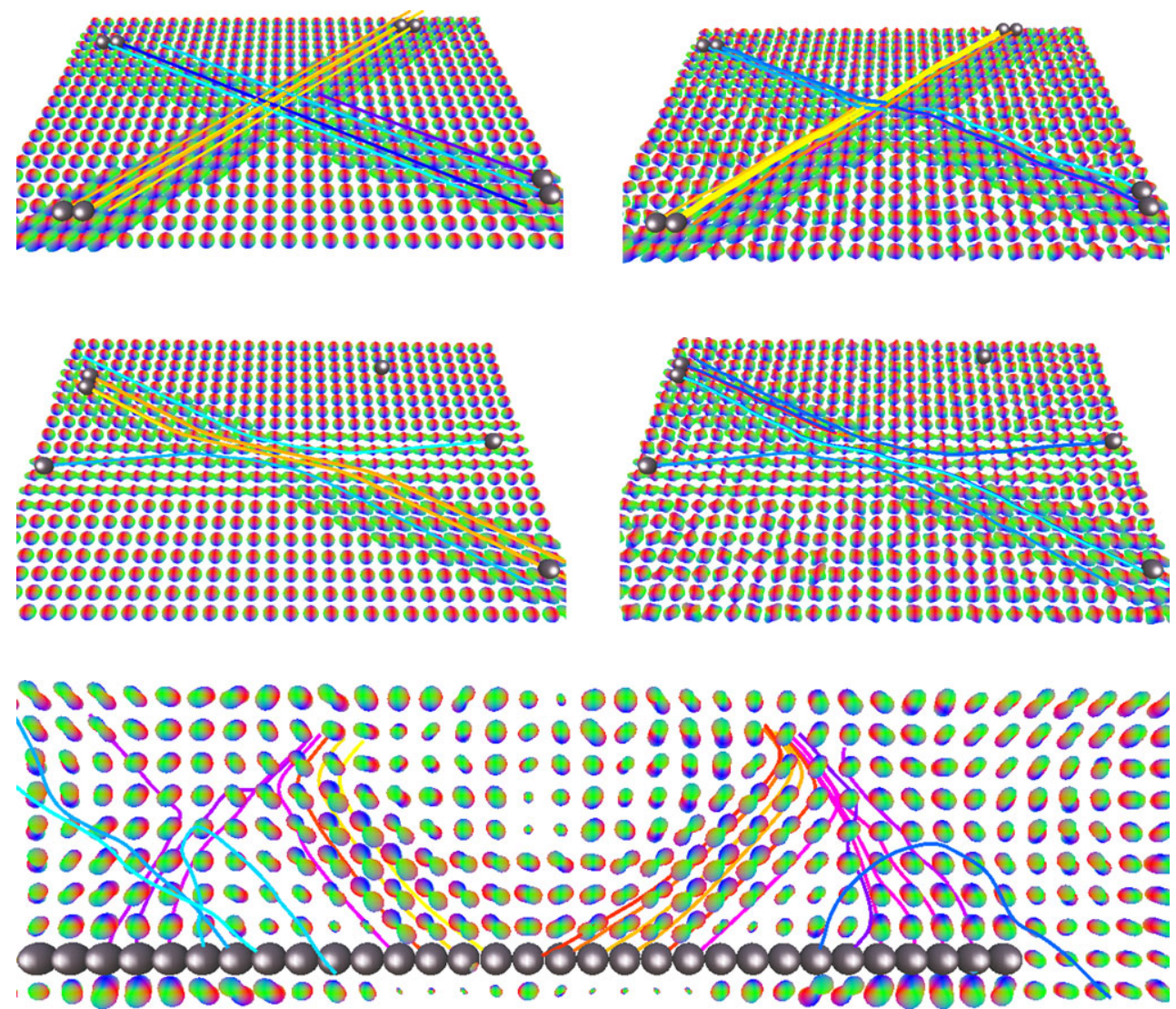

Fig. 5 Second order tensor field on real data and DTI-streamlines with black seed points. In DTI, intersecting fibers are impossible since there is only one tangent vector occupying a point. Apparent crossings are due to the projection of spatial curves to image plane

\section{Experimental Results}

\subsection{Simulated Data}

We computed Finsler streamlines in tensor fields that simulate two fiber bundles crossing at angles of 30 and 65 degrees. The tensors in the crossing area are generated using the Gaussian mixture model [37] assuming signal with $b$ value $1000 \mathrm{~s} / \mathrm{m}^{2}$. Noisy images were generated by simulating Rician noise with SNR around 15, in our case this corresponds to the inverse of standard deviation of the (Gaussian) noise added to the real and complex parts of the signal. We solved the Finsler streamlines (25) using a third order Runge-Kutta model for integration [22]. If the fractional anisotropy (FA) became less than 0.2 or if the inner product of the tangent of the curve and the local principal eigenvector became less than 0.1 , the tracking stops. These parameters are similar to those used in the standard DTI-fiber tracking. We have colored the fibers using a temperature map according to their connectivity (32) so that fibers with highest (lowest) connectivity are colored red (blue). See Figs. 4 and 3.

\subsection{Real Data}

We used a HARDI scan of a human brain with $b$-value 1000 $\mathrm{s} / \mathrm{m}^{2}$, and 132 gradient directions. As initial points we used
30 voxels in the area where the major fiber bundles called Corpus Callosum and the Corona Radiata are known to cross each other. The parameters used are identical to those used in simulated data except that the critical value of FA was set to 0.09 . For comparison we added also the corresponding second order tensor field and a result of standard DTItracking in Fig. 5. Finsler streamlines in Fig. 6 indeed do show crossings in those regions where this is to be expected, unlike standard DTI-tracking. In Fig. 7 we used otherwise identical settings, but increased the number of initial points.

\subsection{Performance Comparison}

In this section we compare the performance of our method (Finsler) with standard DTI streamline tracking (DTI) and a simple method based on computing the global maximum of the ODF (ODF-gmax), as discussed in Sect. 4.2. In the first experiment we measured the time required by each of these methods to track a streamline of (approximately) equal length. The number of search directions of the (global) maximum is set equal to the number of scanning directions, here 54. The same number of directions is also used by our Finsler tracking method at the initial point/voxel, see Fig. 2. Table 2 shows the performance of each method. As it can be seen, our method performs similarly to standard DTI tracking and is around three times faster than the ODF-gmax method. 
Fig. 6 Fourth order tensor glyphs on real HARDI data and Finsler streamlines with seed points. Although these streamlines are generally not on the same plane, they do actually cross each other in a way that is in accordance with the anatomical knowledge of human brain [25]

Fig. 7 (Color online) A result of Finsler fiber tracking, when using a larger set of initial points. Besides the crossings of Corona Radiata and Corpus Callosum, also some Cingulum fibers are clearly visible. The color of the fibers indicate the major orientation of the fiber. Blue: top-bottom, red: left-right, green: back-front
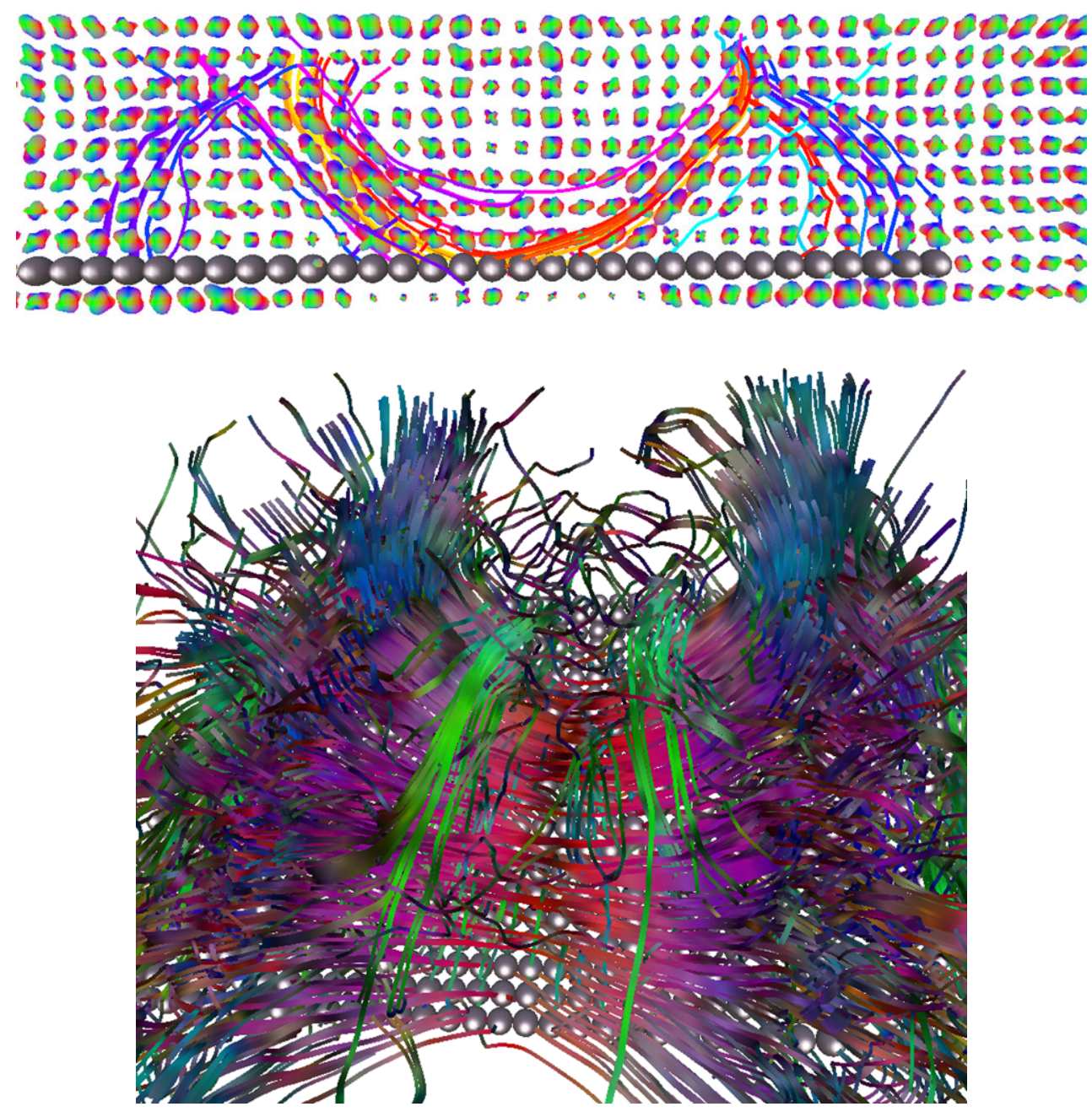

Table 2 Performance of the three methods when tracking a streamline of (approximately) equal length. Third column gives the timing (milliseconds) for each method. The number of search and initial directions is 54

\begin{tabular}{lll}
\hline Method & Euclidean length & Timing \\
\hline DTI & 27.3 & 1.5 \\
Finsler & 27.5 & 1.9 \\
ODF-g max & 27.1 & 6.8 \\
\hline
\end{tabular}

In the next experiment we increased the number of directions (search for ODF-gmax and initial for Finsler) to 250 , see Table 3 . Note that in this case our method is about 8 times faster than the ODF-gmax method. The large drop in performance of the ODF-gmax method can be partly explained by the fact that now five times more evaluations are required for maxima computation. Moreover, evaluating the generalized FA becomes also more expensive.

Finally, tracking streamlines using the data set and initialization from Fig. 6 took 0.08 seconds by the DTI method, 0.2 seconds with our method and 2.8 seconds using ODF-
Table 3 Performance of Finsler and ODF-gmax methods when tracking a streamline of (approximately) equal length. Third column gives the timing (milliseconds) for each method. The number of search and initial directions is 250

\begin{tabular}{lll}
\hline Method & Euclidean length & Timing \\
\hline Finsler & 27.1 & 2.9 \\
ODF-g max & 27.2 & 23.6 \\
\hline
\end{tabular}

gmax. The number of search/initial directions in this experiment was 54.

\section{Summary and Conclusions}

We have presented a method with which one can obtain a regularized orientation distribution function from the tensor components (polynomial coefficients) representing the HARDI signal, with a single matrix multiplication. The method includes a parameter for Laplace-Beltrami regularization, which solves the heat equation on the sphere, and is 
therefore suitable for noisy spherical data such as HARDI. The choice of the order of the tensor approximating the signal has the effect of an additional regularization. This truncation however in practice cannot be avoided and is ultimately bounded by the number of measurements.

We have also shown a novel method for fiber tracking in HARDI case, which is a true generalization of the streamline tracking in the diffusion tensor setting. Our method is based on Finsler geometry and does not require solving for local maxima of the tensors, which typically is a requirement for other methods.

In addition we have introduced a measure for filtering out those streamlines that are not well connected, i.e. that have small overall diffusivity. Our connectivity measure can be applied to any curve in a regular space equipped with a norm.

Acknowledgements The Netherlands Organization for Scientific Research is gratefully acknowledged for financial support. We thank Paulo Rodrigues and Vesna Prčkovska at the Biomedical Image Analysis group of the department of Biomedical Engineering at Eindhoven University of Technology for kindly providing the real and simulated HARDI data.

Open Access This article is distributed under the terms of the Creative Commons Attribution Noncommercial License which permits any noncommercial use, distribution, and reproduction in any medium, provided the original author(s) and source are credited.

\section{Appendix}

Here we show an example of using the formula of Clebsch to project a polynomial of degree $n$ to a harmonic and a non-harmonic part. Applying the projection iteratively one obtains a decomposition of the original polynomial to a sum of harmonic polynomials of degrees up to $n$ [29]. Let $D_{n}(y)$ be a homogeneous polynomial of degree $n$. In our case this is

$D_{n}(y)=D_{i_{1} \cdots i_{n}} y^{i_{1}} \cdots y^{i_{n}}$,

where

$y=(\sin \theta \cos \varphi, \sin \theta \sin \varphi, \cos \theta)$,

i.e. a homogeneous polynomial restricted to the sphere in

$\mathbb{R}^{3}$. The Clebsch projection operator in dimension three is as follows

$\mathbb{P}_{n}:=\sum_{k=1}^{\left[\frac{n}{2}\right]} \frac{(-1)^{k-1}}{4^{k}} \frac{\Gamma\left(n-k+\frac{1}{2}\right)}{k ! \Gamma\left(n+\frac{1}{2}\right)}|y|^{2 k} \triangle^{k}$,

where $\Gamma$ refers to the Gamma function:

$\Gamma(z)=\int_{0}^{\infty} t^{z-1} e^{-t} d t$.
Computing

$H_{n}(y)=D_{n}(y)-\mathbb{P}_{n}\left(D_{n}\right)(y)$,

gives $H_{n}(y)$ which is a homogeneous and harmonic polynomial of degree $n$. For proof see [29]. Next we show a concrete example of computing Laplace-Beltrami regularized ODF by simply multiplying the $n$th order tensor components fitted to the signal by a matrix. For simplicity we show this in degree two, but it extends to all even degrees. Let

$S=\left(S_{11}, S_{12}, S_{13}, S_{22}, S_{23}, S_{33}\right)$

be the six components of the symmetric tensor approximating the DT-MRI signal. Then the local second order polynomial describing the signal is

$$
\begin{aligned}
D_{2}(y)= & S_{11} y^{1} y^{1}+2 S_{12} y^{1} y^{2}+S_{13} y^{1} y^{3} \\
& +S_{22} y^{2} y^{2}+2 S_{23} y^{2} y^{3}+S_{33} y^{3} y^{3},
\end{aligned}
$$

where $y$ is as in (34). Clebsch projection (35) applied to a polynomial of degree two (39) gives

$$
\begin{aligned}
\mathbb{P}\left(D_{2}(y)\right) & =\frac{1}{6}|y|^{2} \triangle D_{2}(y) \\
& =\frac{1}{3}\left(\left(y^{1}\right)^{2}+\left(y^{2}\right)^{2}+\left(y^{3}\right)^{2}\right)\left(S_{11}+S_{22}+S_{33}\right) \\
& =\frac{1}{3}\left(S_{11}+S_{22}+S_{33}\right) \\
& =H_{0}(y)
\end{aligned}
$$

which is the zeroth order solid harmonic. The second order solid harmonic is then the remainder

$$
\begin{aligned}
H_{2}(y)= & D_{2}(y)-\mathbb{P}\left(D_{2}(y)\right) \\
= & \left(\frac{2}{3} S_{11}-\frac{1}{3} S_{22}-\frac{1}{3} S_{33}\right)\left(y^{1}\right)^{2} \\
& +\left(\frac{2}{3} S_{22}-\frac{1}{3} S_{11}-\frac{1}{3} S_{33}\right)\left(y^{2}\right)^{2} \\
& +\left(\frac{2}{3} S_{33}-\frac{1}{3} S_{22}-\frac{1}{3} S_{11}\right)\left(y^{3}\right)^{2} \\
& +2 S_{12} y^{1} y^{2}+2 S_{13} y^{1} y^{3}+2 S_{23} y^{2} y^{3} .
\end{aligned}
$$

Multiplying these harmonic components $H_{0}, H_{2}$ with appropriate terms in (9), i.e. $2 \pi P_{0}(0)=2 \pi$ and $2 \pi P_{2}(0)=$ $-\pi$, we obtain the ODF:

$D_{2}(y)=\pi\left(2 H_{0}(y)-H_{2}(y)\right)$.

To apply Laplace-Beltrami smoothing as in (17), we multiply each harmonic component with the order dependent 
regularization term as follows

$D_{2}(y, \tau)=\pi\left(e^{-\tau(0+1) 0} 2 H_{0}(y)-e^{-\tau(2+1) 2} H_{2}(y)\right)$.

Thus the zeroth order part of the regularized ODF is

$H_{0}(\tau)=\frac{2 \pi}{3}\left(S_{11}+S_{22}+S_{33}\right)$

which can be expanded to second order homogeneous polynomial by taking the Kronecker product of this scalar and identity matrix $I_{3}$ (similarly for higher order polynomials [5]). By doing this we obtain the following representation for $H_{0}(\tau)$ :

$H_{0}(y, \tau)=\frac{2 \pi}{3}\left(S_{11}+S_{22}+S_{33}\right)\left(\left(y^{1}\right)^{2}+\left(y^{2}\right)^{2}+\left(y^{3}\right)^{2}\right)$.

Recall that $\left(\left(y^{1}\right)^{2}+\left(y^{2}\right)^{2}+\left(y^{3}\right)^{2}\right)=1$.

The regularized second order part of the ODF is:

$$
\begin{aligned}
H_{2}(y, \tau)= & -\pi e^{-6 \tau}\left[\left(\frac{2}{3} S_{11}-\frac{1}{3} S_{22}-\frac{1}{3} S_{33}\right)\left(y^{1}\right)^{2}\right. \\
& +\left(\frac{2}{3} S_{22}-\frac{1}{3} S_{11}-\frac{1}{3} S_{33}\right)\left(y^{2}\right)^{2} \\
& +\left(\frac{2}{3} S_{33}-\frac{1}{3} S_{22}-\frac{1}{3} S_{11}\right)\left(y^{3}\right)^{2} \\
& \left.+2 S_{12} y^{1} y^{2}+2 S_{12} y^{1} y^{3}+2 S_{23} y^{2} y^{3}\right]
\end{aligned}
$$

Now, the sum $H_{0}(y, \tau)+H_{2}(y, \tau)$ gives exactly the regularized second order ODF. With some reverse engineering, we can easily determine the transformation matrix that takes signal $S(y)$ to regularized ODF: $H_{0}(y, \tau)+H_{2}(y, \tau)$.

Given a symmetric second order tensor $S(y)$ (38) representing the raw HARDI signal, the $j$ th term in the components vector (cf. (38)) of second order tensor $S_{\mathrm{ODF}}(y, \tau)$ representing $\tau$-regularized ODF is then

$$
S_{\mathrm{ODF}}(y, \tau)^{j}=\sum_{k=1}^{6} 2 \pi c_{j k} S(y)^{k},
$$

where

$$
\begin{aligned}
& c_{11}=\frac{1}{3}\left(1-e^{-6 \tau}\right), \\
& c_{12}=c_{21}=0, \\
& c_{13}=c_{31}=0, \\
& c_{14}=c_{41}=\frac{1}{6} e^{-6 \tau}, \\
& c_{15}=c_{51}=0,
\end{aligned}
$$

$$
\begin{aligned}
& c_{16}=\frac{1}{6} e^{-6 \tau}, \\
& c_{22}=2, \\
& c_{23}=c_{32}=0, \\
& c_{24}=c_{42}=0, \\
& c_{25}=c_{52}=0, \\
& c_{26}=c_{62}=0, \\
& c_{33}=2, \\
& c_{34}=c_{43}=0, \\
& c_{35}=c_{53}=0, \\
& c_{36}=c_{63}=0, \\
& c_{44}=\frac{1}{3}\left(1-e^{-6 \tau}\right), \\
& c_{45}=c_{54}=0, \\
& c_{46}=\frac{1}{6} e^{-6 \tau}, \\
& c_{55}=2, \\
& c_{56}=c_{65}=0, \\
& c_{66}=\frac{1}{3}\left(1-e^{-6 \tau}\right) .
\end{aligned}
$$

This matrix as well as the corresponding higher order tensors have to be computed only once. Thus to model a HARDI ODF with a polynomial of order $n$ with regularization parameter $\tau$, one needs only to fit an $n$th order polynomial to the raw signal, multiply it with a precomputed matrix $c_{i j}$ and assign a parameter $\tau$ of choice.

\section{References}

1. Aganj, I., Lenglet, C., Sapiro, G.: ODF maxima extraction in spherical harmonic representation via analytical search space reduction. In: The Thirteenth International Conference on Medical Image Computing and Computer Assisted Intervention, Beijing, China, pp. 84-91 (2010)

2. Aganj, I., Lenglet, C., Sapiro, G., Yacoub, E., Ugurbil, K., Harel, N.: Reconstruction of the orientation distribution function in single- and multiple-shell q-ball imaging within constant solid angle. Magn. Reson. Med. 64, 554-566 (2010)

3. Anderson, A.W.: Measurement of fiber orientation distributions using high angular resolution diffusion imaging. Magn. Reson. Med. 54(5), 1194-1206 (2005)

4. Arsigny, V., Fillard, P., Pennec, X., Ayache, N.: Geometric means in a novel vector space structure on symmetric positive-definite matrices. SIAM J. Matrix Anal. Appl. 29(1), 328-347 (2006)

5. Astola, L., Florack, L.: Finsler geometry on higher order tensor fields and applications to high angular resolution diffusion imaging. Int. J. Comput. Vis. (2010). doi:10.1007/s11263-010-0377-Z

6. Astola, L., Fuster, A., Florack, L.: A Riemannian scalar measure for diffusion tensor images. Pattern Recognit. (2010). doi:10.1016/j.patcog.2010.09.009 
7. Astola, L., Florack, L., ter Haar Romeny, B.: Measures for pathway analysis in brain white matter using diffusion tensor images. In: Information Processing in Medical Imaging: 20th International Conference (IPMI 2007), Kerkrade, The Netherlands. Information Processing in Medical Imaging, vol. 20, pp. 642-649. Springer, Berlin (2007)

8. Bao, D., Chern, S.-S., Shen, Z.: An Introduction to RiemannFinsler Geometry. Springer, Berlin (2000)

9. Barmpoutis, A., Hwang, M., Howland, D., Forder, J., Vemuri, B.: Regularized positive-definite fourth order tensor field estimation from DW-MRI. Neuroimage 45(1), 152-162 (2009)

10. Bulow, T.: Spherical diffusion for 3D surface smoothing. IEEE Trans. Pattern Anal. Mach. Intell. 26(12), 1650-1654 (2004)

11. Callaghan, P.T.: Principles of Nuclear Magnetic Resonance Microscopy. Oxford Science, Oxford (1991)

12. Chern, S.S., Chen, W.H., Lam, K.S.: Lectures on Differential Geometry. World Scientific, New Jersey (1999)

13. Deriche, R., Descoteaux, M.: Splitting tracking through crossing fibers: multidirectional Q-ball tracking. In: Proceedings of the 4th International Symposium on Biomedical Imaging: From Nano to Macro (ISBI'07), Arlington, Virginia, USA, vol. 4 (2007)

14. Descoteaux, M.: High angular resolution diffusion MRI: from local estimation to segmentation and tractography. $\mathrm{PhD}$ thesis, University of Nice-Sophia Antipolis, Nice, France (2008)

15. Descoteaux, M., Angelino, E., Fitzgibbons, S., Deriche, R.: Regularized, fast and robust analytical Q-ball imaging. Magn. Reson. Med. 58, 497-510 (2007)

16. Florack, L.: Codomain scale space and regularization for high angular resolution diffusion imaging. In: CVPR Workshop on Tensors in Image Processing and Computer Vision, Anchorage, Alaska, The United States. CVPR, vol. 20. Springer, Berlin (2008)

17. Florack, L., Astola, L.: A multi-resolution framework for diffusion tensor images. In: CVPR Workshop on Tensors in Image Processing and Computer Vision, Anchorage, Alaska, The United States. CVPR, vol. 20. Springer, Berlin (2008)

18. Florack, L., Balmashnova, E.: Decomposition of high angular resolution diffusion images into a sum of self-similar polynomials on the sphere. In: Proceedings of the Eighteenth International Conference on Computer Graphics and Vision, GraphiCon'2008, June 2008 (invited paper)

19. Florack, L., Balmashnova, E.: Two canonical representations for regularized high angular resolution diffusion imaging. In: MICCAI Workshop on Computational Diffusion MRI, New York, USA, September, pp. 94-105 (2008)

20. Ghosh, A., Moakher, M., Deriche, R.: Ternary quartic approach for positive 4th order diffusion tensors revisited. In: Proceedings of IEEE International Symposium on Biomedical Imaging (ISBI), Boston, Massachusetts, USA, pp. 618-621 (2009)

21. Ghosh, A., Descoteaux, M., Deriche, R.: Riemannian framework for estimating symmetric positive definite 4th order diffusion tensors. In: Proceedings of 11th International Conference on Medical Image Computing and Computer Assisted Intervention (MICCAI), New York, USA, pp. 858-865 (2008)

22. Gottlieb, S., Shu, C.: Total variation diminishing Runge-Kutta schemes. Math. Comput. 67(221), 73-85 (1998)

23. Hess, C., Mukherjee, P., Han, E., Xu, D., Vigneron, D.: Q-ball reconstruction of multimodal fiber orientations using the spherical harmonic basis. Magn. Reson. Med. 56(1), 104-117 (2006)

24. Jian, B., Vemuri, B., Özarslan, E., Carney, P., Mareci, T.: A novel tensor distribution model for the diffusion-weighted MR signal. NeuroImage 37, 164-176 (2007)

25. Kiernan, J.A.: Barr's the Human Nervous System. Wolters Kluwer Health, 9th edn. (2008)

26. Kuhnel, W.: Differential Geometry, Curves-Surfaces-Manifolds. Student Mathematical Library, vol. 16. American Mathematical Society, Providence (2002). Translated from the German edition
27. Melonakos, J., Pichon, E., Angenent, S., Tannenbaum, A.: Finsler active contours. IEEE Trans. Pattern Anal. Mach. Intell. 30(3), 412-423 (2008)

28. Mori, S., Crain, B.J., Chacko, V.P., van Zijl, P.C.: Threedimensional tracking of axonal projections in the brain by magnetic resonance imaging. Ann. Neurol. 45(2), 265-269 (1999)

29. Müller, C. (ed.): Analysis of Spherical Symmetries in Euclidean Spaces. Applied Mathematical Sciences, vol. 129. Springer, New York (1998)

30. O'Donnell, L., Haker, S., Westin, C.: New approaches to estimation of white matter connectivity in diffusion tensor MRI, elliptic PDEs and geodesics in a tensor-warped space. In: Proceedings of Medical Imaging, Computing and Computer Assisted Intervention. Lecture Notes in Computer Science, vol. 2488, pp. 459-466. Springer, Berlin (2002)

31. Prados, E., Soatto, S., Lenglet, C., Pons, J.-P., Wotawa, N., Deriche, R., Faugeras, O.: Control Theory and Fast Marching Techniques for Brain Connectivity Mapping, vol. 1, pp. 1076-1083 (2006)

32. Qi, L., Yu, G., Wu, E.: Higher order positive semidefinite diffusion tensor imaging. SIAM J. Imaging Sci. 3(3), 416-433 (2010)

33. Shen, Z.: Lectures on Finsler Geometry. World Scientific, Singapore (2001)

34. Tristán-Vega, A., Westin, C.-F., Aja-Fernándes, S.: Estimation of fiber orientation probability density functions in high angular resolution diffusion imaging. NeuroImage 18, 638-650 (2009)

35. Tuch, D.: Diffusion MRI of complex tissue structure. PhD-thesis, Massachusetts Institute of Technology, USA, January 2002

36. Tuch, D.: Q-ball imaging. Magn. Reson. Med. 52(4), 577-582 (2002)

37. Tuch, D., Reese, T., Wiegell, M., Makris, N., Belliveau, J., van Wedeen, J.: High angular resolution diffusion imaging reveals intravoxel white matter fiber heterogeneity. Magn. Reson. Med. 48(6), 1358-1372 (2004)

38. Özarslan, E., Mareci, T.: Generalized diffusion tensor imaging and analytical relationships between diffusion tensor imaging and high angular resolution diffusion imaging. Magn. Reson. Med. 50, 955-965 (2003)

39. Özarslan, E., Shepherd, T., Vemuri, B., Blackband, S., Mareci, T.: Resolution of complex tissue microarchitecture using the diffusion orientation transform (DOT). NeuroImage 31(3), 1086-1103 (2006)

40. Özarslan, E., Vemuri, B., Mareci, T.: Generalized scalar measures for diffusion MRI using trace, variance and entropy. Magn. Reson. Med. 53(4), 866-876 (2005)

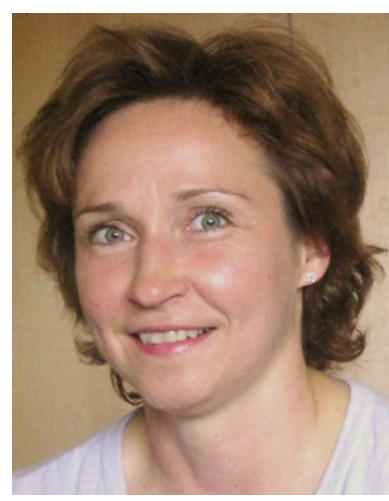

Laura Astola received her M.Sc. degree in mathematics at Helsinki University, Finland, in 2000. In 2009, she obtained her degree of Licentiate of Engineering from Helsinki University of Technology. She received her Ph.D. degree with a thesis on differential geometry and applications in medical image analysis from Eindhoven University of Technology in 2010. She is currently a postdoctoral fellow at Biometrics in Wageningen University and Research Center and Netherlands Consortium for Systems Biology. Her research concerns reconstruction and inference of metabolic and genetic networks using statistical analysis and techniques from pattern recognition and graph theory. 


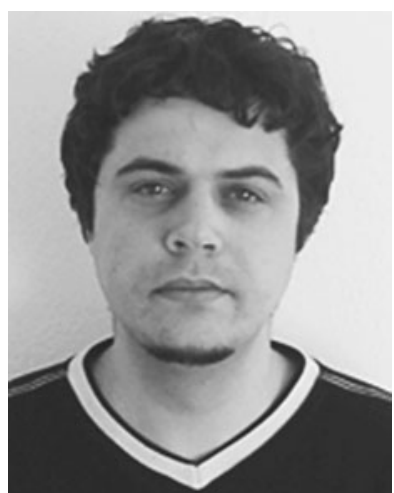

Andrei Jalba received the B.Sc. (1998) and M.Sc. (1999) degrees in applied electronics and information engineering from the "Politehnica" University of Bucharest, Romania. He holds a Ph.D. in mathematics and natural sciences (2004) from the University of Groningen, The Netherlands. Currently he is an assistant professor in Visualization at the Eindhoven University of Technology. His research interests include scientific and parallel computing with applications in (medical) image processing and computer graphics.

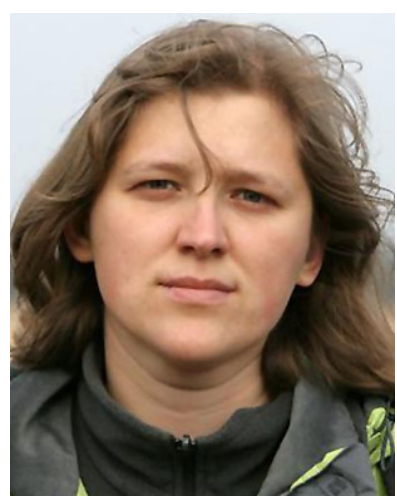

Evgeniya Balmashnova received her M.Sc. degree in mathematics at Novosibirsk State University, Russia, in 2000. In 2001-2003 she followed postgraduate program Mathematics for Industry at the Stan Ackermans Institute in Eindhoven. She received her Ph.D. degree in 2007 from Eindhoven University of Technology. She is currently a postdoctoral fellow at the Department of Mathematics and Computer Science at the same university. Her research is focused on medical image analysis, high angular resolution diffusion imaging and diffusion tensor imaging in particular, multiscale approaches in image analysis.

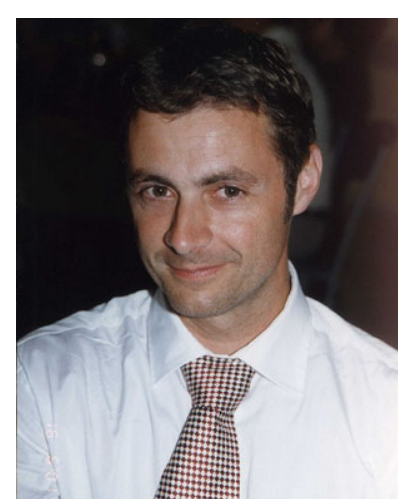

Luc Florack received his M.Sc. degree in theoretical physics in 1989, and his Ph.D. degree cum laude in 1993 with a thesis on image structure under the supervision of professor Max Viergever and professor Jan Koenderink, both from Utrecht University, The Netherlands. During the period 19941995 he was an ERCIM/HCM research fellow at INRIA SophiaAntipolis, France, with professor Olivier Faugeras, and at INESC Aveiro, Portugal, with professor Antonio Sousa Pereira. In 1996 he was an assistant research professor at DIKU, Copenhagen, Denmark, with professor Peter Johansen, on a grant from the Danish Research Council. In 1997 he returned to Utrecht University, were he became an assistant research professor at the Department of Mathematics and Computer Science. In 2001 he moved to Eindhoven University of Technology, Department of Biomedical Engineering, were he became an associate professor in 2002. In 2007 he was appointed full professor at the Department of Mathematics and Computer Science, and established the chair of Mathematical Image Analysis, but retained a parttime professorship at the former department. His research covers mathematical models of structural aspects of signals, images, and movies, particularly multiscale and differential geometric representations and their applications, with a focus on complex magnetic resonance images for cardiological and neurological applications. In 2010, with support of the Executive Board of Eindhoven University of Technology, he established the Imaging Science \& Technology research group (IST/e), a cross-divisional collaboration involving several academic groups on image acquisition, biomedical and mathematical image analysis, visualization and visual analytics. 